\title{
ALGEBRAS OF BOUNDED FINITE DIMENSIONAL REPRESENTATION TYPE
}

\author{
by ALLEN D. BELL and K. R. GOODEARL $\dagger$
}

(Received 15 February, 1994)

1. Introduction and background. It is well known that for finite dimensional algebras, "bounded representation type" implies "finite representation type"; this is the assertion of the First Brauer-Thrall Conjecture (hereafter referred to as Brauer-Thrall I), proved by Roiter [26] (see also [23]). More precisely, it states that if $R$ is a finite dimensional algebra over a field $k$, such that there is a finite upper bound on the $k$-dimensions of the finite dimensional indecomposable right $R$-modules, then up to isomorphism $R$ has only finitely many (finite dimensional) indecomposable right modules. The hypothesis and conclusion are of course left-right symmetric in this situation, because of the duality between finite dimensional left and right $R$-modules, given by $\operatorname{Hom}_{k}(-, k)$. Furthermore, it follows from finite representation type that all indecomposable $R$ modules are finite dimensional [25].

For an arbitrary $k$-algebra $R$, the assumption of a finite upper bound on the $k$-dimensions of the finite dimensional indecomposable (right) $R$-modules should perhaps be termed "bounded finite dimensional representation type". It is natural to ask whether this assumption leads to finite representation type for the finite dimensional (right) $R$-modules, but without any restriction on $R$ counterexamples are abundant. For instance, if $R$ is the ring of all eventually constant sequences of elements from $k$, then one easily sees that all finite dimensional $R$-modules are semisimple (whence all finite dimensional indecomposable $R$-modules are simple), and that all simple $R$-modules are onedimensional over $k$, whereas $R$ has infinitely many isomorphism types of simple modules.

On the other hand, our main results show that if $R$ is either finitely generated as a $k$-algebra (Section 2), or noetherian as a ring (Section 3 ), then bounded finite dimensional representation type does indeed imply that $R$ has only finitely many isomorphism types of finite dimensional indecomposable modules. In order to gain information on infinite dimensional representations, we also investigate analogous boundedness conditions in terms of composition series length instead of vector space dimension (Section 4). For a large class of noetherian rings $R$, including those that satisfy a polynomial identity, it turns out that the existence of a finite upper bound on the lengths of the finite-length indecomposable (right) $R$-modules implies that $R$ has only finitely many isomorphism types of finite-length indecomposable modules.

This research was motivated by discussions with R. Farnsteiner, who pointed out to us that any nonzero finite dimensional Lie algebra over a field has finite dimensional indecomposable representations of arbitrarily large dimension and asked whether such a conclusion can be obtained from ring-theoretic results alone. The Lie algebra result just stated is due to Zassenhaus in positive characteristic [32, Theorem 7; 33, p. 2, lines 14-17]; in characteristic zero it is an easy consequence of standard representation theory. Zassenhaus actually proved a stronger existence result for indecomposable representations of a finite dimensional Lie algebra $g$ over a field of positive characteristic. He

$\dagger$ The research of the second author was partially supported by a National Science Foundation grant.

Glasgow Math. J. 37 (1995) 289-302. 
showed that if $M$ is any maximal ideal of the center of the enveloping algebra $U(g)$, then there exist indecomposable finite dimensional representations of $g$, with arbitrarily large dimension, all composition factors of which are annihilated by maximal ideals of $U(g)$ that contract to $M$ [ibid].

One ring-theoretic result along these lines, guaranteeing a somewhat weaker finiteness property of the representation type, is already known. This is a theorem of Farkas [8], stating that if $R$ is a finitely generated algebra over a field $k$, such that all finite dimensional right $R$-modules are semisimple, then $R$ has only finitely many isomorphism types of $n$-dimensional right modules for each positive integer $n$. The hypothesis that all finite dimensional right $R$-modules are semisimple may be rephrased as the assumption that all finite dimensional right $R$-modules have Loewy length at most 1 . This led us to investigate how severely it restricts the representation type of $R$ to assume a finite upper bound just on the Loewy lengths of the finite dimensional indecomposable right $R$-modules. It turns out that such a bound places restrictions in particular on the representation type of the semisimple $R$-modules. In fact, we obtain a generalization of Farkas's theorem, namely that the existence of a finite upper bound on the Loewy lengths of the finite dimensional indecomposable right $R$-modules implies that $R$ has only finitely many isomorphism types of $n$-dimensional semisimple right modules for each positive integer $n$. This theorem holds for noetherian $k$-algebras as well as for finitely generated $k$-algebras.

Definitions. The socle series (sometimes called the lower Loewy series) for a module $A$ is the series of submodules

$$
\operatorname{soc}^{0}(A)=0 \leq \operatorname{soc}^{1}(A)=\operatorname{soc}(A) \leq \operatorname{soc}^{2}(A) \leq \ldots,
$$

where $\operatorname{soc}^{i+1}(A) / \operatorname{soc}^{i}(A)$ is the socle of $A / \operatorname{soc}^{i}(A)$. In case $\operatorname{soc}^{m}(A)=A$ for some $m$, the least such $m$ is called the Loewy length of $A$. In other cases the Loewy length of $A$ is customarily not defined.

When we refer to the length of a module, we mean its composition series length.

Note that if $A$ is a module over a ring $R$ such that $R$ modulo the Jacobson radical $J(R)$ is semisimple (completely reducible), then the Loewy length of $A$ (if defined) equals the least power of $J(R)$ that annihilates $A$. We observe that if $A$ is a module and $d$ is a positive integer such that all cyclic submodules of $A$ have Loewy length that is bounded by $d$, then $A$ has Loewy length and the Loewy length of $A$ is at most $d$. (One has only to note that each cyclic submodule $B$ of $A$ satisfies $B=\operatorname{soc}^{d}(B) \subseteq \operatorname{soc}^{d}(A)$.)

We show in Sections 2 and 3 that if $R$ is either finitely generated as a $k$-algebra or noetherian as a ring, then the existence of a finite bound on the Loewy lengths [respectively, lengths] of finite dimensional indecomposable (right) $R$-modules implies that there are only finitely many isomorphism types of semisimple [respectively, arbitrary] $n$-dimensional $R$-modules for each positive integer $n$.

Definition. A ring $R$ is said to have finite representation type provided $R$ is right artinian and there are only finitely many isomorphism types of finitely generated indecomposable right $R$-modules. This condition was shown to be left-right symmetric by Eisenbud and Griffith [7, Theorem 1.2].

Ringel and Tachikawa proved that if $R$ has finite representation type, then all 
$R$-modules are direct sums of finitely generated modules [25, Corollary 4.4]. In particular, it follows that all indecomposable $R$-modules are finitely generated.

The classical version of Brauer-Thrall $I$ was generalized to artinian rings by Auslander, who proved that if $R$ is a right artinian ring and there is a finite upper bound on the lengths of all finitely generated indecomposable right $R$-modules, then $R$ has finite representation type ([1, Theorem 3.1; Theorem 4.4]; see also [23, pp. 109, 124]).

In Section 4 we show, for example, that if $R$ is a noetherian ring satisfying the second layer condition and if there is a bound on the Loewy lengths of all finitely generated indecomposable right $R$-modules with co-artinian annihilators, then $R$ is isomorphic to the direct product of an artinian ring and a noetherian ring having no nonzero modules with co-artinian annihilators. If there is a bound on the lengths of all finitely generated indecomposable right $R$-modules with co-artinian annihilators, then the artinian factor has finite representation type. We note that this result fails if $R$ does not satisfy the second layer condition. We actually prove results of greater generality involving modules whose annihilators come from a specified set of co-artinian ideals.

There is an alternative approach to some of our key technical results concerning PI-rings using idempotent ideals and a result of Small and Robson. We discuss this briefly in Section 5.

2. Bounded finite dimensional representation type for affine algebras. Throughout this section, let $k$ denote a field. Recall that an affine algebra over $k$ is any finitely generated $k$-algebra. When discussing $k$-algebras and their modules, we reserve the adjective "finite dimensional" to mean finite dimensional as a vector space over $k$. We remark that the functors $\operatorname{Hom}_{k}(-, k)$ provide dimension-preserving dualities between the categories of finite dimensional right and left modules over any $k$-algebra $R$. Thus for example a bound on the Loewy lengths, the lengths, or the dimensions of the finite dimensional indecomposable right $R$-modules will also be a bound for the corresponding left $R$-modules. Likewise, the number of isomorphism types of finite dimensional indecomposable $R$-modules is the same on the left as on the right, and all finite dimensional right $R$-modules are semisimple if and only if this is true on the left.

We start by investigating affine $k$-algebras for which there is a finite upper bound on the Loewy lengths of the finite dimensional indecomposable right modules. This is the same as placing a bound on the Loewy lengths of arbitrary finite dimensional right modules, since the Loewy length of a finite direct sum of modules is just the maximum of the Loewy lengths of the summands. Moreover, it would suffice to place a bound on the Loewy lengths of finite dimensional uniform cyclic modules, since any finite dimensional module can be embedded in a finite direct sum of finite dimensional uniform modules, and since the Loewy length of any module equals the supremum of the Loewy lengths of its cyclic submodules.

The proof of the following proposition is an adaptation of Farkas's proof in [8] to a more general situation.

Proposition 2.1. Let $R$ be an affine k-algebra, and assume that the Loewy lengths of all finite dimensional right $R$-modules are bounded by a positive integer $d$. If $R$ satisfies a polynomial identity, then $R$ is finite dimensional over $k$. 
Proof. We first prove the proposition for semiprime algebras $R$. Since $R$ satisfies the ACC on semiprime ideals [28, Theorem 4.5.7; 18, 13.10.7], we may assume by noetherian induction that $R$ is infinite dimensional but every semiprime factor ring of $R$ is finite dimensional. The ACC implies that $R$ has only finitely many minimal primes, so the induction hypothesis easily yields that $R$ is prime. As all proper prime factor rings of $R$ are artinian, $R$ has Krull dimension at most 1 . If $R$ were artinian, then $R$ would be finite dimensional over $k$ by [29, Theorem 28; 18, Theorem 13.10.3]. Thus $R$ has Krull dimension exactly 1 . It follows from [29, Theorem 35 and proof] that $R$ is noetherian.

Let $I$ be a semiprime ideal of $R$ : we will show that $I^{d}=I^{d+1}$. Plainly we may assume $I \neq 0$, in which case $R / I$ is finite dimensional. It follows that $R / I^{d+1}$ is finite dimensional (since $R$ is noetherian) with Jacobson radical $I / I^{d+1}$. Since the Loewy length of the module $R / I^{d+1}$ is at most $d$, this implies that $\left(R / I^{d+1}\right) I^{d}=0$, and so $I^{d}=I^{d+1}$.

Let $n$ be the PI-degree of $R$ and let $P$ be a maximal ideal of $R$ such that $R / P$ has PI-degree $n$ (such a $P$ exists since $R$ is semiprimitive - use, e.g., [18, Lemma 13.7.2]). If we let $X=Z(R) \backslash P$, then by [29, Theorem 19; 18, Proposition 13.7.5], $R X^{-1}$ has Jacobson radical $P X^{-1}$. Since $P^{d}=P^{d+1}$, we see that $\left(P X^{-1}\right)^{d}=\left(P X^{-1}\right)^{d+1}$. By Nakayama's Lemma we have $P X^{-1}=0$, and as $X$ consists of regular elements, this shows that $P=0$. Since $R$ is not artinian, this is impossible. This contradiction proves the proposition in the semiprime case.

Now suppose that $R$ is not semiprime and let $N$ be the prime radical of $R$. By the above $R / N$ is finite dimensional. By [4, p. 14, Proposition 2.8], $N$ is finitely generated as a right $R$-module. It now follows that each power $N^{l}$ is finitely generated as a right $R$-module, and so each module $N^{l-1} / N^{l}$ is finite dimensional. Thus each factor $R / N^{l}$ is finite dimensional. By [2, Theorem 2.3], $N$ is nilpotent, and the result follows.

A slight generalization of the weaker version of Zassenhaus's result follows; for a generalization of the stronger form, see Corollary 4.5.

COROLlaRY 2.2. Suppose that $\mathbf{g}$ is a nonzero finite dimensional Lie algebra over $k$, and that char $k>0$. Then $\mathbf{g}$ has finite dimensional indecomposable representations with arbitrarily large Loewy length, and hence with arbitrarily large $k$-dimension.

Proof. Since $U(\mathbf{g})$ is an infinite dimensional affine PI-algebra, Proposition 2.1 implies that there cannot be a bound on the Loewy lengths.

The first conclusion of Corollary 2.2 of course fails for semisimple Lie algebras in characteristic 0 , because of Weyl's Theorem, which states that in this case all nonzero finite dimensional representations have Loewy length 1.

Corollary 2.2 can also be derived from a result of Hochschild [10, Theorem 2], which states that if $I$ is the augmentation ideal of the center $Z$ of $U(\mathbf{g})$ (that is, $I=Z \cap \mathbf{g} U(\mathbf{g})$ ), then $\bigcap_{n=1}^{\infty} U(\mathbf{g}) I^{n}=0$. Since $U(\mathbf{g})$ is finitely generated as a $Z$-module [12], $U(\mathbf{g}) / U(\mathbf{g}) I^{n}$ is finite dimensional over $k$ for each $n$, and so it follows from Hochschild's result that $U(\mathbf{g})>U(\mathbf{g}) I>U(\mathbf{g}) I^{2}>\ldots$ Now $I$ must annihilate each irreducible $U(\mathbf{g}) / U(\mathbf{g}) I^{n}$. module, from which we see that $U(\mathbf{g}) / U(\mathbf{g}) I^{n}$ has Loewy length at least $n$. Therefore some indecomposable direct summand of $U(\mathbf{g}) / U(\mathbf{g}) I^{n}$ must have Loewy length at least $n$.

The group-theoretic analog of Corollary 2.2 is the following (cf. [22, Theorem 5.3.7; Corollary 5.3.10]). Let $G$ be an infinite, finitely generated group. If either $G$ is 
abelian-by-finite, or $k$ has characteristic $p>0$ and $G$ is $p$-abelian-by-finite, then $G$ has finite dimensional indecomposable representations with arbitrarily large Loewy length.

To apply Proposition 2.1 to arbitrary affine $k$-algebras, we follow the reduction used by Farkas [8]; namely, we factor out the intersection of the annihilators of all the modules of a given finite dimension, which results in a PI-algebra.

Proposition 2.3. Let $R$ be an affine $k$-algebra.

(a) If the Loewy lengths of all finite dimensional right $R$-modules are bounded by $a$ positive integer $d$, then $R$ has only finitely many isomorphism types of $n$-dimensional semisimple right (or left) modules for each $n \in \mathbb{N}$.

(b) If the lengths of all finite dimensional indecomposable right $R$-modules are bounded by a positive integer $d$, then $R$ has only finitely many isomorphism types of $n$-dimensional right (or left) modules for each $n \in \mathbb{N}$.

Proof. Define a set $\mathscr{A}$ of right $R$-modules in each of the two parts as follows:

$$
\begin{gathered}
\mathscr{A}=\left\{A_{R} \mid A \text { is simple and } \operatorname{dim}_{k} A \leq n\right\} ; \\
\mathscr{A}=\left\{A_{R} \mid A \text { is indecomposable and } \operatorname{dim}_{k} A \leq n\right\} ;
\end{gathered}
$$

and let $I=\bigcap\left\{\operatorname{ann}_{R}(A) \mid A \in \mathscr{A}\right\}$. For each $A \in \mathscr{A}$, the ring $R / \operatorname{ann}_{R}(A)$ embeds in the matrix ring $M_{l}(k)$ for $l=\operatorname{dim}_{k} A \leq n$. Consequently, $R / I$ satisfies the identities of $n \times n$ matrices and so is a PI-algebra.

Moreover, in both cases the Loewy lengths of all finite dimensional $(R / I)$-modules are bounded by $d$. Thus by Proposition $2.1, R / I$ is finite dimensional. In part (a), $R / I$ is semisimple artinian, while in part (b), Brauer-Thrall I implies that $R / I$ has finite representation type. (Note that the existence of a bound on the lengths of the finite dimensional indecomposable right $(R / I)$-modules implies that the $k$-dimensions of these modules are also bounded.) In either case, we see that $\mathscr{A}$ contains only finitely many isomorphism types, and the desired conclusion follows.

COROLlary 2.4. (Farkas) Let $R$ be an affine $k$-algebra, and assume that all finite dimensional right $R$-modules are semisimple. Then $R$ has only finitely many isomorphism types of $n$-dimensional right (or left) modules for each $n \in \mathbb{N}$.

THEOREM 2.5. Let $R$ be an affine $k$-algebra, and assume that the $k$-dimensions of all finite dimensional indecomposable right $R$-modules are bounded by a positive integer $d$. Then $R$ has only finitely many isomorphism types of finite dimensional indecomposable right (or left) modules. In fact, there is an idempotent ideal $I \subseteq R$ (not necessarily proper) with finite codimension such that I annihilates all finite dimensional right or left $R$-modules and $R / I$ has finite representation type.

Proof. Let $I$ be the intersection of the annihilators of all finite dimensional indecomposable right $R$-modules. As in the proof of Proposition $2.3(\mathrm{~b}), R / I$ is finite dimensional and has finite representation type. Moreover, $I$ annihilates all finite dimensional right $R$-modules. It follows from [4, p. 14, Proposition 2.8] that $R / I^{2}$ is also finite dimensional, and so it is annihilated by $I$. Hence, $I=I^{2}$. 
The duality discussed at the beginning of this section shows that $I$ annihilates all finite dimensional left $R$-modules as well, completing the proof.

Under the hypotheses of Theorem 2.5, we cannot conclude that $R$ itself is finite dimensional. For example, the Weyl algebra $A_{1}(\mathbb{C})=\mathbb{C}\{x, y\} /\langle y x-x y-1\rangle$ is an affine $\mathbb{C}$-algebra with no nonzero finite dimensional modules. A more interesting example is provided by the "usual idealizer" inside $A_{1}(\mathbb{C})$, namely the ring $R=\mathbb{C}+y A_{1}(\mathbb{C})$. This ring is an affine noetherian $\mathbb{C}$-algebra [24, Proposition 2], the ideal $I=y A_{1}(\mathbb{C})$ is the unique proper nonzero ideal of $R$, all finite dimensional $R$-modules are annihilated by $I$, and thus all finite dimensional indecomposable $R$-modules are one-dimensional. This example also shows that the ideal $I$ in Theorem 2.5 need not be a direct summand of $R$.

We remark that Roseblade [27, Theorem A] has shown that if $k$ is an algebraic extension of a finite field and $G$ is a polycyclic-by-finite group, then all simple modules over the group algebra $k[G]$ are finite dimensional. The algebra $k[G]$ is affine and noetherian; furthermore, we may choose $G$ such that $k[G]$ does not satisfy a polynomial identity and so that there are simple modules of arbitrarily large finite dimension (for example, choose $G$ to be a non-abelian poly- $\mathbb{Z}$ group). Irving [11] has given an example of an affine non-noetherian algebra over an arbitrary field $k$ such that all simple modules are finite dimensional and there are simple modules of arbitrarily large finite dimension. We are unaware of an affine noetherian example of this type over a field of characteristic zero. Using the main result of [2] and the concept of PI-degree, it is not hard to see that if $R$ is an affine algebra over an algebraically closed field, then $R$ satisfies a polynomial identity if and only if $J(R)$ is nilpotent and there is a finite bound on the dimensions of the simple right $R$-modules.

3. Bounded finite dimensional representation type for noetherian algebras. We continue to assume in this section that $k$ denotes a field. The results of Section 2 all have analogs for noetherian $k$-algebras, but we have to allow for noetherian PI-algebras over $k$ that have no nonzero modules which are finite dimensional over $k$. For example, any infinite dimensional field extension of $k$ has these properties.

Proposition 3.1. Let $R$ be a noetherian $k$-algebra, and assume that the Loewy lengths of all finite dimensional right $R$-modules are bounded by a positive integer $d$. If $R$ satisfies $a$ polynomial identity, then there is a direct product decomposition $R=R_{1} \times R_{2}$ such that $R_{1}$ is a finite dimensional $k$-algebra while $R_{2}$ is a noetherian $k$-algebra having no nonzero right or left modules of finite $k$-dimension.

We shall derive Proposition 3.1 from more general results in the following section. As consequences, we immediately obtain the following results using the methods of Section 2.

PROPOSITION 3.2. Let $R$ be a noetherian $k$-algebra.

(a) If the Loewy lengths of all finite dimensional right $R$-modules are bounded by a positive integer $d$, then $R$ has only finitely many isomorphism types of $n$-dimensional semisimple right (or left) modules for each $n \in \mathbb{N}$. 
(b) If the lengths of all finite dimensional indecomposable right $R$-modules are bounded by a positive integer $d$, then $R$ has only finitely many isomorphism types of $n$-dimensional right (or left) modules for each $n \in \mathbb{N}$.

COROLlaRY 3.3. Let $R$ be a noetherian k-algebra, and assume that all finite dimensional right $R$-modules are semisimple. Then $R$ has only finitely many isomorphism types of $n$-dimensional right (or left) modules for each $n \in \mathbb{N}$.

THEOREM 3.4. Let $R$ be a noetherian $k$-algebra, and assume that the $k$-dimensions of all finite dimensional indecomposable right $R$-modules are bounded by a positive integer $d$. Then $R$ has only finitely many isomorphism types of finite dimensional indecomposable right (or left) modules. In fact, there is an idempotent ideal $I \subseteq R$ (not necessarily proper) with finite codimension such that $I$ annihilates all finite dimensional right or left $R$-modules and $R / I$ has finite representation type.

4. Bounded finite-length representation type for noetherian rings. The existence of noetherian non-artinian V-rings (i.e., rings all of whose simple modules are injective) shows that a bound on the lengths of finite-length indecomposable modules over a noetherian ring will not in general imply that the ring is artinian. There exist simple noetherian V-rings with only one isomorphism type of simple modules [5, Theorem 1.4], and there also exist simple noetherian V-rings with infinitely many isomorphism types of simple modules [21, Example (a)]. The latter example shows that a finite bound on the Loewy lengths of the finite-length modules over a noetherian ring (or even a bound on the lengths of the indecomposable finite-length modules) does not always imply that there are only finitely many isomorphism types of finite-length indecomposable modules.

In order to obtain positive results, we restrict ourselves to modules with co-artinian annihilators, and we apply ideas from the localization theory of noetherian rings (cf. $[15,9]$ for more details on the following definitions and a proof of the first lemma).

Definition. Let $P$ and $Q$ be prime ideals in a noetherian ring $R$. There exists a link from $P$ to $Q$, denoted $P \leadsto Q$, if and only if there exists an ideal $A$ lying between $P \cap Q$ and $P Q$ such that the $(R / P, R / Q)$-bimodule $(P \cap Q) / A$ is nonzero and torsionfree on each side. For co-artinian maximal ideals $P$ and $Q$, the definition simplifies considerably: namely, $P \leadsto Q$ if and only if $P \cap Q \neq P Q$.

Links between co-artinian maximal ideals arise from non-split extensions of the corresponding simple modules, as the following easy lemma shows.

Lemma 4.1. Let $P$ and $Q$ be co-artinian maximal ideals in a noetherian ring $R$, and let $A$ and $B$ be simple right $R$-modules such that $A P=0$ and $B Q=0$. Then $P \leadsto Q$ if and only if there exists a non-split extension $0 \rightarrow B \rightarrow C \rightarrow A \rightarrow 0$.

Definitions. Let $\mathcal{M}$ be a nonempty set of co-artinian maximal ideals in a noetherian ring $R$. 
(a) The set $\mathcal{M}$ is right (left) link-closed provided $\mathcal{M}$ is closed under incoming (outgoing) links.

(b) The set $\mathcal{M}$ is right (left) link-finite provided that for each $P \in \mathcal{M}$, there are at most finitely many prime ideals of $R$ linked to (from) $P$.

(c) The set $\mathcal{M}$ satisfies the right second layer condition provided that for each $P \in \mathcal{M}$, if we let $E$ be the injective hull $E\left((R / P)_{R}\right)$, then there is no prime ideal strictly smaller than $P$ that is an assassinator prime of $E / \operatorname{ann}_{E}(P)$.

(d) An $R$-module $A$ is classically $M$-primary provided each element of $A$ is annihilated by some product of ideals from $\mathcal{M}$.

Several comments about the preceding definitions are in order.

(i) Conditions (b) and (c) hold automatically in case $R$ is a PI-ring [19, Theorem 7; 20, Theorem 7; 15, Theorem A.2.4], in case $R$ is the enveloping algebra of a solvable finite dimensional Lie algebra $[14$, p. $61 ; 15$, Theorems A.3.2, A.3.9], or in case $R$ is the group algebra of a polycyclic-by-finite group $[3$, Theorem $6.4 ; 13$, Proposition $10 ; 14$, Theorem 4.5; 15, Theorems A.4.6, A.4.7].

(ii) Among noetherian rings in general, only one example is known where condition (c) holds and condition (b) fails [31, Theorem 4.4].

(iii) Conditions (a) and (b) are really conditions on co-artinian maximal ideals, since any prime ideal linked to or from a co-artinian prime must itself be co-artinian. This follows from Lenagan's Theorem [17, Proposition; 18, Theorem 4.1.6], that says that a noetherian bimodule which is artinian on one side must also be artinian on the other.

(iv) Lenagan's Theorem also shows that the set of all co-artinian maximal ideals in $R$ is right and left link-closed. Clearly if $R$ is an algebra over a field, then the set of all maximal ideals of $R$ with finite codimension is right and left link-closed.

(v) Conditions (a)-(c) pass easily to factor rings. In particular, if $\mathcal{M}$ is a set of maximal ideals satisfying any of these conditions and $I$ is an ideal of $R$, then the collection $\mathscr{M}^{\prime}=\{P / I \mid P \in \mathscr{M}$ and $P \supseteq I\}$ is a set of maximal ideals of $R / I$ satisfying the same condition(s). To see this for (a) and (b), note that it follows immediately from the definition of a link that if $P$ and $Q$ are prime ideals of $R$ containing $I$ for which $P / I \leadsto Q / I$, then $P \leadsto Q$. Concerning (c), note that for any prime ideal $P$ of $R$ containing $I$, the $(R / I)$-module injective hull of $R / P$ equals the annihilator of $I$ in the $R$-module injective hull of $R / P$.

In the next three results, it suffices to bound the Loewy lengths of uniform cyclic modules satisfying the given condition. (See the remarks before Proposition 2.1.)

THEOREM 4.2. Let $R$ be a noetherian ring, and let $M$ be a right link-closed, right link-finite set of co-artinian maximal ideals of $R$, satisfying the right second layer condition. Assume that the Loewy lengths of all classically $\mathcal{M}$-primary right $R$-modules are bounded by a positive integer $d$.

(a) All the prime ideals in $\mathcal{M}$ are minimal primes, and so $\mathcal{M}$ is finite.

(b) The ideal $J=\bigcap \mathcal{M}$ satisfies $J^{d}=J^{d+1}$, and all classically $\mathcal{M}$-primary right or left $R$-modules are annihilated by $J^{d}$.

(c) The ring $R / J^{d}$ is artinian and all classically M-primary left $R$-modules have Loewy length at most $d$.

(d) If $\mathcal{M}$ is also left link-closed, then $J^{d}$ is a ring direct summand of $R$, and so $R \cong\left(R / J^{d}\right) \times R_{2}$ for some ring $R_{2}$. 
Proof. (a) Assume that $P \in \mathcal{M}$ is not minimal and let $Q$ be a prime ideal of $R$ properly contained in $P$. Set $\mathcal{M}^{\prime}=\left\{P^{\prime} \in \mathcal{M} \mid Q \subseteq P^{\prime}\right\}$. By comment (v) above, the image of $\mathcal{M}^{\prime}$ in $R / Q$ is right link-closed, right link-finite, and satisfies the right second layer condition, and clearly $d$ is a bound on the Loewy lengths of the classically $\mathcal{M}^{\prime}$-primary right $(R / Q)$-modules. Thus the hypotheses remain in force if we pass to $R / Q$ and replace $\mathcal{M}$ by $\left\{P^{\prime} / Q \mid P^{\prime} \in \mathcal{M}^{\prime}\right\}$.

Hence we may assume that $R$ is prime but not artinian. Let $P \in \mathcal{M}$, let $S$ be a simple right $(R / P)$-module, and let $E$ be the injective hull of $S$ over $R$. Since $\mathcal{M}$ is right link-closed and satisfies the right second layer condition, it is a standard consequence of Jategaonkar's Main Lemma (see [15, Corollary 9.1.3; 9, Theorem 11.6]) that the factors $\operatorname{soc}^{i} E / \operatorname{soc}^{i-1} E$ are direct sums of simple modules whose annihilators are in $\mu$; the link-finiteness of $\mathcal{M}$ implies that only finitely many such annihilators can occur for each $i$. Consequently, each $\operatorname{soc}^{i} E$ is annihilated by a finite product of primes from $\mathcal{M}$ and so is unfaithful $(0 \notin \mathcal{M})$. It is also the case that $E$ is the union of its socle series $([15$, Corollary 9.1.3]), and so $E$ is classically $\mathcal{M}$-primary. Thus the hypotheses imply that $E$ has Loewy length at most $d$, and so $E=\operatorname{soc}^{d} E$. However over a prime right noetherian ring any nonzero injective module is faithful (cf. [9, Lemma 12.9]). This contradiction proves the minimality of the elements of $M$.

(b) and (c) Let $J=\bigcap \mathcal{M}$. The right $R$-module $R / J^{d+1}$ is classically $\mathcal{M}$-primary, and so its Loewy length is at most $d$. It follows from this that $J^{d}=J^{d+1}$, and hence that any finitely generated right or left $R$-module that is annihilated by a product of elements of $M$ is annihilated by $J^{d}$. Thus $d$ is also a bound on the Loewy lengths of such left $R$-modules.

(d) We first show that $\operatorname{Hom}_{R}\left(J^{d}, E(A)\right)=0$ for any classically $\mathcal{M}$-primary right $R$-module $A$. It suffices to consider the case that $A$ is a simple right $(R / P)$-module for some $P \in \mathscr{M}$; hence, it follows as in part (a) that $E(A)$ is classically $\mathcal{M}$-primary. Now if $\operatorname{Hom}_{R}\left(J^{d}, E(A)\right) \neq 0$, then we can replace $E(A)$ with a finitely generated submodule, and we can then replace that submodule with a simple factor $B$; hence we would have $\operatorname{Hom}_{R}\left(J^{d}, B\right) \neq 0$ for a classically $\mathcal{M}$-primary simple right $R$-module $B$. Such a module $B$ must be annihilated by some $Q \in \mathcal{M}$, and this implies that $J^{d} Q<J^{d}$, contrary to (b). Thus the claim follows.

Now let $K=\mathrm{r}$-ann $\left(J^{d}\right)$. If $J^{d}+K \neq R$, then there is a maximal ideal $P$ which contains $J^{d}+K$ and hence both $J$ and $K$. As $P$ contains $J$, we must have $P \in \mathcal{M}$. Since $J^{d}$ is a noetherian bimodule, $R / K$ embeds as a right $R$-module in a finite direct sum of copies of $J^{d}$. By the last paragraph, we must have $\operatorname{Hom}_{R}(R / K, E(A))=0$ for all classically $\mathcal{M}$-primary right $R$-modules $A$. Thus we must have $\operatorname{Hom}_{R}(R / K, R / P)=0$, contradicting the fact that $K \subseteq P$. Hence, $R=J^{d}+K$.

Set $K^{\prime}=J^{d} \cap K$. Since $J^{d} K=0$, we see that $K$, and hence also $K^{\prime}$, is left artinian. Thus by Lenagan's Theorem, $K^{\prime}$ is right artinian. Now by [9, Corollary 7.6], $K^{\prime}$ is annihilated on the right by a product of primes $Q_{i}$ such that for each $i$, there is an ideal link from a prime in $\mathcal{M}$ to $Q_{i}$. Since $\mathcal{M}$ consists of minimal primes, it satisfies both the left and right second layer conditions, and so by [15, Theorem 8.2.4], all the primes $Q_{i}$ are in the left link closure of $\mathcal{M}$, and hence in $\mathcal{M}$. Thus either r-ann $\left(K^{\prime}\right)=R$ or some ideal in $\mathcal{M}$ contains $\mathrm{r}$-ann $\left(K^{\prime}\right) \supseteq K$; as in the last paragraph the latter possibility cannot occur. Thus we have $K^{\prime}=0$, which proves that $R=J^{d} \oplus K$.

The idealizer example at the end of Section 2 shows that both (a) and (d) of Theorem 
4.2 can fail without the second layer condition. The necessity of the extra hypothesis in (d) can already be seen in the algebra $R=\left(\begin{array}{ll}k & k \\ 0 & k\end{array}\right)$, if $\mathcal{M}$ consists just of the prime $P=$ $\left(\begin{array}{ll}0 & k \\ 0 & k\end{array}\right)$. Note that in this example, all classically $\mathcal{M}$-primary $R$-modules are semisimple. As one consequence of Theorem 4.2, we obtain the following analog of Proposition 2.1 .

COROLlary 4.3. Let $R$ be a fully bounded noetherian ring. If the Loewy lengths of all finite-length right $R$-modules are bounded by a positive integer $d$, then $R$ is artinian.

Proof. All primitive ideals of $R$ are co-artinian by [9, Proposition 8.4]. Hence, if $\mathcal{M}$ is the set of maximal ideals of $R$, then $\cap M=J(R)$, the finitely generated classically $\mathcal{M}$-primary $R$-modules are precisely the $R$-modules of finite length, and $\mathcal{M}$ is right and left link-closed by Lenagan's Theorem. Furthermore, $\mathcal{M}$ satisfies the right and left second layer conditions by [15, Proposition 8.1.1], and $\mathscr{M}$ is link-finite by [15, Theorem 6.2.16]. Thus by Theorem 4.2, $\mathcal{M}$ is finite and $J(R)^{d}$ is a ring direct summand of $R$. Since $J(R)$ contains no nonzero idempotents, $J(R)^{d}=0$, and therefore we conclude that $R$ is artinian.

COROLlaRY 4.4. Let $R$ be a noetherian ring which is module-finite over a subring $C$ of its center. Let $M$ be a maximal ideal of $C$, and let $M$ be the set of those maximal ideals of $R$ that contract to $M$. If the Loewy lengths of all classically $M$-primary right $R$-modules are bounded by a positive integer $d$, then $M^{d}=M^{d+1}$ and $M^{d} R$ is a ring direct summand of $R$.

Proof. The hypotheses imply that $R$ is a PI-ring and that $C$ is noetherian [6, Theorem 1]. Since the localization $C_{M}$ embeds in the localization $R_{M}$, it follows from Nakayama's Lemma that $M R \neq R$, and consequently the set $\mathcal{M}$ is nonempty. Moreover, $\mathcal{M}$ is finite because it corresponds to the set of maximal ideals of the finite dimensional algebra $R / M R$, and $\mathcal{M}$ is link-closed by [15, Theorem A.7.2; 9, Theorem 11.20]. Now Theorem 4.2 applies: if $J=\cap M$, then $J^{d}=J^{d+1}$ and $J^{d}$ is a ring direct summand of $R$. Clearly $M R \subseteq J$, and $J / M R$ is the radical of $R / M R$, whence $J / M R$ is nilpotent. It follows that $M^{d} R=M^{d+1} R=J^{d}$; in particular, $M^{d} R$ is a ring direct summand of $R$. Finally, a second application of Nakayama's Lemma shows that $M^{d}=M^{d+1}$.

We can now give the following generalization of the sharp form of Zassenhaus's existence theorem for finite dimensional indecomposable representations of Lie algebras in characteristic $p$.

CoRollary 4.5. Suppose that $\mathbf{g}$ is a nonzero finite dimensional Lie algebra over $k$, and that char $k>0$. Let $M$ be a maximal ideal of the center of $U(\mathbf{g})$, and let $M$ be the set of those maximal ideals of $U(\mathbf{g})$ that contract to $M$. Then $\mathbf{g}$ has finite dimensional indecomposable classically M-primary representations with arbitrarily large Loewy length, and hence with arbitrarily large $k$-dimension.

Proof. It is well-known that $U(\mathrm{~g})$ is noetherian, and in [12] it is shown that $U(\mathrm{~g})$ is module-finite over its center. Since $U(\mathbf{g})$ is a domain and $M^{d} U(\mathbf{g})$ is a proper nonzero 
ideal for each positive integer $d$, the ideals $M^{d} U(\mathrm{~g})$ cannot be ring direct summands of $U(\mathrm{~g})$. Thus by Corollary 4.4 there cannot be a bound on the Loewy lengths of the finite dimensional classically $\mathcal{M}$-primary $U(\mathbf{g})$-modules.

Proof of Proposition 3.1. Let $\mathcal{M}$ be the set of maximal ideals of $R$ of finite codimension and note that $\mathcal{M}$ is right and left link-closed. Since $R$ is a PI-algebra, the conclusion of Proposition $4.2(\mathrm{~d})$ is valid. As the finite dimensional $R$-modules are precisely the classically $\mathcal{M}$-primary finitely generated $R$-modules, this proves Proposition 3.1 .

We can now prove the analogous results with bounds on length and dimension instead of Loewy length. Note that any right link-closed set of maximal ideals in a noetherian PI-ring satisfies the hypotheses in the first sentence of the next result.

THEOREM 4.6. Let $R$ be a noetherian ring, and let $\mathcal{M}$ be a right link-closed set of co-artinian maximal ideals of $R$, satisfying the right second layer condition. Suppose that the lengths of all finitely generated indecomposable classically $M$-primary right $R$-modules are bounded by a positive integer $d$.

(a) All the prime ideals in $\mathcal{M}$ are minimal primes, and so $\mathcal{M}$ is finite.

(b) The ideal $J=\bigcap \mathcal{M}$ satisfies $J^{d}=J^{d+1}$, and all classically $\mathcal{M}$-primary right or left $R$-modules are annihilated by $J^{d}$.

(c) The ring $R / J^{d}$ is artinian and has finite representation type.

(d) If $\mathcal{M}$ is also left link-closed, then $J^{d}$ is a ring direct summand of $R$, and so $R \cong\left(R / J^{d}\right) \times R_{2}$ for some ring $R_{2}$.

Proof. All the hypotheses of Theorem 4.2 are satisfied, except possibly right link-finiteness. Suppose that there are $d$ distinct prime ideals $P_{1}, \ldots, P_{d}$ in $R$ which are all linked to a prime $Q$ in $\mathcal{M}$. Since $\mathcal{M}$ is right link-closed, each $P_{i} \in \mathcal{M}$. Now let $A_{1}, \ldots, A_{d}, B$ be simple right $R$-modules such that $A_{i} P_{i}=0$ for $i=1, \ldots, d$ and $B Q=0$. By Lemma 4.1 , there exist non-split extensions of $B$ by each $A_{i}$, and so each $A_{i}$ embeds in $E(B) / B$. Hence $E(B)$ has a submodule $C$ such that $C \supset B$ and $C / B \cong A_{1} \oplus \ldots \oplus A_{d}$. But then $C$ is a finitely generated indecomposable classically $M$-primary right $R$-module of length $d+1$, contrary to our hypotheses. This shows that at most $d-1$ primes of $R$ can be linked to any one prime in $\mathcal{M}$. Statements (a), (b), (d) now follow from Theorem 4.2. Observe that $R / J^{d}$ is artinian, and that the classically $\mathcal{M}$-primary $R$-modules are precisely the $\left(R / J^{d}\right)$-modules. Since there is a bound on the lengths of the finitely generated indecomposable right $\left(R / J^{d}\right)$-modules, Auslander's generalization of Brauer-Thrall I $\left(\left[\mathbf{1} ; \mathbf{2 3}\right.\right.$, p. 124]) shows that $R / J^{d}$ has finite representation type. Thus (c) is proved.

COROLLARY 4.7. Let $R$ be a noetherian ring which is module-finite over a subring $C$ of its center. Let $M$ be a maximal ideal of $C$, and let $M$ be the set of those maximal ideals of $R$ that contract to $M$. If the lengths of all finitely generated indecomposable classically $M$-primary right $R$-modules are bounded by a positive integer $d$, then $M^{d}=M^{d+1}$ and $R / M^{d} R$ has finite representation type. Moreover $M^{d} R$ is a ring direct summand of $R$.

COROLlaRy 4.8. Let $R$ be a noetherian algebra over a field $k$, and let $\mathcal{M}$ be a right link-closed set of finite-codimension maximal ideals of $R$. Suppose that the $k$-dimensions of all finite dimensional indecomposable classically $\mathcal{M}$-primary right $R$-modules are bounded 
by a positive integer $d$. Then there is an idempotent ideal $I \subseteq R$ with finite codimension such that I annihilates all classically M-primary right or left $R$-modules and $R / I$ has finite representation type.

For the sake of completeness, we mention a result of Jøndrup [16, Theorem 1 and Proposition 3]. If $R$ is either a PI-ring or a right noetherian ring in which all right primitive ideals are co-artinian, and if $R$ has only finitely many isomorphism types of cyclic indecomposable right modules, then $R$ must be right artinian.

5. Idempotent ideals. In this section we sketch an alternative approach to some of the material in Sections 2 and 3, using idempotent ideals. This approach was pointed out to us by L. W. Small.

We begin with a slight generalization of a result of Small and Robson.

Proposition 5.1. If $R$ is a PI-ring which either satisfies the ACC on ideals or is affine as an algebra over a commutative noetherian ring, then $R$ has only finitely many idempotent ideals.

Proof. If $R$ satisfies the ACC on ideals, this is just [30, Theorem 3]. If $R$ is an affine algebra over a commutative noetherian ring, the proof in [30, Theorem 4] that $R$ has only finitely many idempotent prime ideals can be extended to the general case using [2, Theorem 2.3].

Recall that a filter of ideals in a ring $R$ is a non-empty collection $\mathscr{I}$ of ideals closed under finite intersections, such that any ideal containing an ideal from $\mathscr{I}$ is also a member of $\mathscr{I}$.

PROPOSITION 5.2. Let $I$ be a filter of co-artinian ideals of a ring $R$ that is closed under products, and suppose that $I$ contains only finitely many idempotent ideals. If there is a bound $d$ on the Loewy lengths of modules annihilated by ideals in $\mathscr{I}$, then there is a (unique) smallest ideal in $\mathscr{I}$; this ideal is idempotent.

Proof. If $P$ is a semiprime ideal in $\mathscr{I}$, the bound on the Loewy lengths implies that $P^{n}=P^{d}$ for all $n \geq d$, and so $P^{d}$ is idempotent. Since $P^{d} \neq Q^{d}$ for distinct semiprime ideals $P, Q$, there must be only finitely many semiprime ideals in $\mathscr{I}$. Since $\mathscr{I}$ is closed under intersections, there is a smallest semiprime ideal $J \in \mathscr{I}$. For any $I \in \mathscr{I}$, the radical of $R / I$ is nilpotent and so $I$ contains a power of a semiprime ideal from $\mathscr{I}$. It follows that $I \supseteq J^{d}$, and so $J^{d}$ is the smallest ideal in $\mathscr{Y}$.

Remark 5.3. If $R$ is a noetherian ring, the set of co-artinian ideals of $R$ is a filter and is closed under products. If $R$ is a noetherian or affine algebra over a field, the set of ideals of finite codimension of $R$ is a filter and is closed under products. (To see this in the affine case, use $[4$, p. 14, Proposition 2.8].)

We now use Propositions 5.1 and 5.2 to derive Proposition 2.1 and weakened versions of Proposition 3.1 and Theorem 4.2. These suffice to give most of the applications in Sections 2 and 3.

COROLLARY 5.4. Let $R$ be an affine algebra over a field $k$, and assume that the Loewy lengths of all finite dimensional right $R$-modules are bounded by a positive integer $d$. If $R$ satisfies a polynomial identity, then $R$ is finite dimensional over $k$. 
Proof. By Propositions 5.1, 5.2 and Remark 5.3, $R$ has a smallest ideal $I$ of finite codimension; and $I$ is idempotent. Since $R$ is an affine PI-algebra, $I$ is contained in every primitive ideal of $R$, and so $I \subseteq J(R)$. By [2, Theorem 2.3; abstract], $J(R)$ is nilpotent, and so $I=0$.

COROLLARY 5.5. Let $R$ be a noetherian ring satisfying a polynomial identity.

(a) If the Loewy lengths of all right $R$-modules with co-artinian annihilators are bounded by a positive integer $d$, then there is an idempotent co-artinian ideal I which annihilates all (right or left) $R$-modules with co-artinian annihilator.

(b) If $R$ is an algebra over a field and the Loewy lengths of all finite dimensional right $R$-modules are bounded by a positive integer $d$, then there is an idempotent ideal I of finite codimension that annihilates all finite dimensional (right or left) $R$-modules.

Proof. These results are immediate from Propositions 5.1, 5.2 and Remark 5.3.

For either of the ideals $I$ described in Corollary 5.5, $R=I \oplus \mathrm{r}$-ann $(I)$. The proof of this fact seems to require extra work, such as that in the proof of Theorem 4.2.

\section{REFERENCES}

1. M. Auslander, Representation theory of Artin algebras, II, Comm. Algebra 1 (1974), 269-310.

2. A. Braun, The nilpotency of the radical in a finitely generated PI ring, J. Algebra 89 (1984), 375-396.

3. K. A. Brown, The structure of modules over polycyclic groups, Math. Proc. Cambridge Phil. Soc. 89 (1981), 257-283.

4. P. M. Cohn, Free associative algebras, Bull. London Math. Soc. 1 (1969), 1-39.

5. J. H. Cozzens, Homological properties of the ring of differential polynomials, Bull. Amer. Math. Soc. 76 (1970), 75-79.

6. D. Eisenbud, Subrings of Artinian and Noetherian rings, Math. Ann. 185 (1970), 247-249. $109-121$.

7. D. Eisenbud and P. Griffith, The structure of serial rings, Pacific J. Math. 36 (1971),

8. D. R. Farkas, Semisimple representations and affine rings, Proc. Amer. Math. Soc. 101 (1987), 237-238.

9. K. R. Goodearl and R. B. Warfield, Jr., An introduction to noncommutative Noetherian rings (Cambridge Univ. Press, 1989).

10. G. Hochschild, An addition to Ado's theorem, Proc. Amer. Math. Soc. 17 (1966), 531-533.

11. R. S. Irving, Affine algebras with any set of integers as the dimensions of simple modules, Bull. London Math. Soc. 17 (1985), 243-247.

12. N. Jacobson, A note on Lie algebras of characteristic p, Amer. J. Math. 74 (1952), 357-359.

13. A. V. Jategaonkar, Noetherian bimodules, in Proc. Conf. on Noetherian Rings and Rings with Polynomial Identities (Durham Univ. 1979), 158-169.

14. A. V. Jategaonkar, Solvable Lie algebras, polycyclic-by-finite groups, and bimodule Krull dimension, Comm. Algebra 10 (1982), 19-69.

15. A. V. Jategaonkar, Localization in Noetherian rings (Cambridge Univ. Press, 1986).

16. S. Jøndrup, Indecomposable modules, in Ring Theory: Proceedings of the 1978 Antwerp Conference ( $F$. Van Oystaeyen, ed.), (Dekker, New York, 1979), 97-104.

17. T. H. Lenagan, Artinian ideals in noetherian rings, Proc. Amer. Math. Soc. 51 (1975), 499-500.

18. J. C. McConnell and J. C. Robson, Noncommutative Noetherian rings (Wiley-Interscience, 1987). 
19. B. J. Müller, Twosided localization in Noetherian PI-rings, in Ring Theory Antwerp 1978 (F. Van Oystaeyen, ed.), (Dekker, New York, 1979), 169-190.

20. B. J. Müller, Two-sided localization in Noetherian PI-rings, J. Algebra 63 (1980), 359-373.

21. B. L. Osofsky, On twisted polynomial rings, J. Algebra 18 (1971), 597-607.

22. D. S. Passman, The algebraic structure of group rings (Wiley, 1977).

23. R. S. Pierce, Associative Algebras, (Springer-Verlag, 1982).

24. R. Resco, Affine domains of finite GK-dimension which are right, but not left, Noetherian, Bull. London Math. Soc. 16 (1984), 590-594.

25. C. M. Ringel and H. Tachikawa, QF-3 rings, J. Reine Angew. Math. 272 (1975), 49-72.

26. A. V. Roiter, Unboundedness of the dimensions of the indecomposable representations of an algebra which has infinitely many indecomposable representations, Izv. Akad. Nauk SSSR Ser. Mat. 32 (1968), 1275-1282 (Russian); English transl. in Math. USSR-Izvestija 2 (1968), 1223-1230.

27. J. E. Roseblade, Group rings of polycyclic groups, J. Pure Appl. Algebra 3 (1973), 307-328.

28. L. H. Rowen, Polynomial identities in ring theory (Academic Press, 1980). (1980)

29. L. W. Small, Rings satisfying a polynomial identity, Vorlesungen Univ. Essen, Heft 5

30. L. W. Small and J. C. Robson, Idempotent ideals in P. I. rings, J. London Math. Soc. (2) 14 (1976), 120-122. $381-392$

31. J. T. Stafford, On the ideals of a noetherian ring, Trans. Amer. Math. Soc. 289 (1985),

32. H. Zassenhaus, Representation theory of Lie algebras of characteristic $p$, Bull. Amer. Math. Soc. 60 (1954), 463-469.

33. H. Zassenhaus, The representations of Lie algebras of prime characteristic, Proc. Glasgow Math. Assoc. 2 (1954), 1-36.

Department of Mathematics

UNIVERSITY OF WISCONSIN-MILWAUKEE

Milwaukee

WISCONSIN 53201

U.S.A.

E-mail. adbell@archimedes.math.uwm.edu
Department of Mathematics

UNIVERSITY OF UTAH

Salt Lake City

UTAH 84112

U.S.A.

Current address:

Department of Mathematics

UNIVERSITY OF CALIFORNIA

Santa Barbara

CaLifornia 93106

U.S.A.

E-mail.goodearl@math.ucsb.edu 